\section{Rücktritt der FMH-Mitglieder} der Assessment-Kommission [1]

Es handelt sich offensichtlich um einen ernsthaften Konflikt, der nicht gelöst werden konnte und in einem Debakel endete. In einer solchen Situation ist Transparenz unerlässlich: Wir Mitglieder der FMH haben ein Recht zu erfahren, was geschehen ist, und uns gegebenenfalls dazu $\mathrm{zu}$ äussern. Und anderseits ist die FMH als demokratische Organisation darauf angewiesen, dass die Basis ihren Teil der Mitverantwortung wahrnehmen kann. Unterbleibt die Kommunikation zwischen Spitze und Basis, droht der FMH Gefahr.

Seit Erscheinen der Rücktrittsmeldung der drei Mitglieder Ende Januar 2004 ist gut ein Monat vergangen. Ich warte auf eine Information von seiten der FMH in der SÄZ. Nun ist es möglich, dass eine solche erfolgt ist und ich sie übersehen habe. Andernfalls aber bitte ich den Zentralvorstand, uns zu orientieren, Stellung zu beziehen und den drei zurückgetretenen Mitgliedern unter Aufhebung allfälliger Sanktionsdrohungen - die Möglichkeit zur Gegendarstellung einzuräumen.

Rudolf Bleuler, Präsident der psychiatrisch tätigen Ärztinnen und Ärzte beider Appenzell und der Schweizerischen Gesellschaft für Intensive Dynamische Kurzpsychotherapie

1 Brandenberg JE, Fischer U, Meister B. Rücktritt der FMH-Mitglieder der Assessment-Kommission. Schweiz Ärztezeitung 2004;85(4):153.

\section{Replik}

Der Zentralvorstand gibt Schreiben, die an ihn gerichtet werden, nicht an Dritte weiter und bezieht dazu auch nicht öffentlich Stellung. Es steht jedermann frei, sich bei den ehemaligen Mitgliedern der Assessment-Kommission direkt zu informieren. Nach Kenntnis des ZV wurden die Rücktrittsschreiben per E-Mail einem grossen Kreis von FMH-Mitgliedern zugänglich gemacht. Es dürfte ein leichtes sein, sich diese bei den Verfassern zu beschaffen. Im übrigen sind die drei Sitze in der Assessment-Kommission interimistisch besetzt worden; die Ärztekammer vom 29. April 2004 wird definitiv über die Vertretung bestimmen. Auch dies wurde einem weiten Kreis von FMH-Mitgliedern und dann in der Schweizerischen Ärztezeitung publiziert.

Reto Steiner, Abt. Kommunikation FMH
Worin liegt das Unbehagen?

Replik auf den Leserbrief von Dr. med.

\section{Armin Oberle}

In seinem Leserbrief äussert Armin Oberle sein grosses, wohl von vielen geteiltes Unbehagen gegenüber der schwierigen, für ihn hoffnungslosen Situation von zerebral schwerstgeschädigten Patienten [1]. Er wirft der SAMW und der Gesellschaft vor, trotz Richtlinien zur Behandlung diese Menschen in einer menschenunwürdigen Situation zu belassen. Er fordert, dieses Leben abzubrechen.

Es ist unüblich, dass die SAMW ihre Richtlinien nach deren Publikation rechtfertigt, wenn andere Meinungen zu den ethisch-juristischen höchst komplexen Fragen geäussert werden. Der Leserbrief von Kollege Oberle mag aber dazu dienen, gewisse Aspekte zu erläutern und zu klären. In den Richtlinien wird klar festgehalten, dass der Wille des Patienten zu respektieren sei und lebenserhaltende Therapien und pflegerische Massnahmen, wie z. B. Ernährung und Flüssigkeitszufuhr, nicht gegen den Willen der Patienten ausgeführt werden dürfen. Liegt keine klare Willensäusserung vor, so ist eine umfassende Pflege weiterhin geboten. Erst bei Komplikationen oder zusätzlichen Ereignissen kann im Rahmen der palliativen Massnahmen von einer weiteren Therapie abgesehen werden.

Die Kommission hat sich die Mühe genommen, den Begriff «schwerstgeschädigt» möglichst präzise zu definieren. Gerade bei den neurodegenerativen Krankheiten kann der Verlauf leider sehr protrahiert sein, und der von Oberle zitierte Verlust der kommunikativen und anderen Fähigkeiten ist langsam, gleitend. Für ihn werden diese Personen in einem menschenunwürdigen Zustand gehalten. $\mathrm{Zu}$ welchem Zeitpunkt im Krankheitsverlauf geht nun das menschenwürdige Dasein verloren? Ist dieses Statement nicht gerade ein Vorwurf und eine Abwertung der Personen, die sich der sehr anspruchsvollen Pflege dieser Patienten widmen?

Die mehrfach zitierte Kategorie Menschenwürde ist für ein Individuum wohl nicht nur aus seinen kognitiven Fähigkeiten abzuleiten. Auch eine Person im persistierenden vegetativen Zustand hat ihre Würde nicht verloren und ist nicht zum «Ding» geworden, wie dies im Leserbrief, wohl unbeabsichtigt, aber implizit suggeriert wird. So ist auch der Vergleich mit dem Schwangerschaftsabbruch so nicht zulässig. Natürlich spielen ökonomische Überlegungen eine Rolle. Die Belastung trifft vor allem die Patienten, weniger die Allgemeinheit, sind diese Patienten doch meist in Pflegeheimen. Das mögliche «Einsparpotential» ist in keinem Verhältnis zu den damit verbundenen ethisch-moralischen Fragen. 
Die Richtlinien haben den bestehenden gesetzlichen Rahmen zu berücksichtigen. Damit zeichnen die Möglichkeiten der palliativen Medizin und der «passiven Sterbehilfe» - die nota bene keineswegs einen kleinen Bereich ausmachen den einzuschlagenden Weg vor.

Wenn der Gesetzgeber die Tötung bestimmter Kranker auch ohne deren explizite Zustimmung erlauben würde, stellt sich doch die Frage, wer diesen Entscheid zu fällen und auszuführen hat. Eine neutrale Beurteilung gibt es bei diesen Fragen nicht. Vielmehr leiden wir alle unter der schwer zu ertragenden Hilflosigkeit angesichts dieser Zustände und der Tatsache, dass nicht alles Leiden vermieden werden kann. Der Wunsch, die Situation zu «kontrollieren», bleibt unerfüllt. Der «Illusion von Kontrolle» darf das Leben der Betroffenen nicht geopfert werden.

Prof. Hannes Stähelin, Basel Präsident der von der Zentralen Ethikkommission der SAMW eingesetzten Subkommission "Behandlung und Betreuung von zerebral schwerst geschädigten Langzeitpatienten»

1 Oberle A. Medizinisch-ethische Richtlinien der SAMW zur Behandlung und Betreuung von zerebral schwerstgeschädigten Langzeitpatienten. Schweiz Ärztezeitung 2004;85(8):377.

\section{Duplik}

Herr Prof. Stähelin findet es unüblich, Richtlinien der SAMW rechtfertigen zu müssen, nachdem sie publiziert worden sind.

Aber auch nach «Roma locuta, causa finita» sollte dem staunenden Volk, das ja nicht in die Vernehmlassung einbezogen worden ist, doch noch die Möglichkeit belassen werden, bescheiden Fragen zu stellen. Um so mehr, als die Erfahrung zeigt, dass solche Richtlinien zeit- und erkenntnisabhängig immer wieder einmal revidiert werden müssen.

Für das Anliegen selber führt seine Antwort auch nicht viel weiter. Es wird bereits als richtig und unumstösslich dargestellt, worüber Zweifel bestehen dürfen und wofür eine Begründung gesucht wird.

Ich fragte nach Angemessenheit und ethischer Schuldung einer an sich nur noch sinnlosen Pflegeleistung und dies ausdrücklich im Fall des bewusstlosen, permanent - also dauernd - vegetativen Status eines Patienten und nicht, wie zitiert, bei «gleitendem Verlust der Kommunikation und anderer Fähigkeiten».
Der Hinweis allein auf «Ethik» ist ebenfalls nicht sehr hilfreich, solange diese nicht begründet werden kann. Ethik als die Lehre von der «Gewohnheit», der «Sitte» ist kein Naturgesetz, hat variable Aussagen, gar von Volk zu Volk verschieden, nur schon in unserer westlichen Welt. So finden christliche Amerikaner die Todesstrafe nicht als unmenschlich, und Niederländer scheinen weniger Bedenken zu haben, Patienten in desolatem Zustand auch ohne deren Zustimmung zu «erlösen».

Der Hinweis, dass diese Dauerpflegefälle die Allgemeinheit nicht sehr belasten, weil sie meist in Pflegeheimen gehalten werden, scheint etwas leichtfertig. Tatsache ist, dass nur sehr Begüterte einen allfällig jahrelangen grossen finanziellen Aufwand ohne weitere Hilfe erbringen können. Meines Erachtens stellt sich nur eine nicht diskutable Verpflichtung zur Lebenserhaltung unter allen Umständen und Verzicht auf jegliche abkürzende Intervention, nämlich die Annahme, dass wir nicht autonom in unserer Lebensgestaltung, sondern als Spitze der Primaten oder als Kinder Gottes mit besonderer Würde, Wert oder Verpflichtung bedacht worden sind und Gott allein über unser Leben und Sterben befinden kann - eine Ansicht, die wohl kaum alle Ethiker teilen dürften.

Alle übrige «Ethik» ist, wenn auch noch so hartnäckig verteidigt und sublimiert, variable menschliche Fiktion und soll nur einem erträglichen Zusammenleben dienlich sein.

Warum ein Vergleich mit dem Schwangerschaftsabbruch unzulässig sein soll, entgeht mir, es sei denn, reine Phänomenologie und nicht Essenz seien massgebend.

Wenn Herr Stähelin weiter argumentiert «die Richtlinien haben den bestehenden rechtlichen Rahmen zu berücksichtigen», wäre zu ergänzen, dass dieser rechtliche Rahmen auch nicht für ewig in Stein gehauen, sondern im Laufe der Menschheitsgeschichte immer wieder nach Bedarf und neuer Sicht ausgeweitet oder eingeengt worden ist. Und gerade mit Bezug auf Medizin und Haltung ihrer Vertreter in Fragen der Menschenbetreuung ist die Schweizerische Akademie der Medizinischen Wissenschaften schliesslich auch wegleitend oder anregend für eine entsprechende Gesetzgebung, weil ihr Einsicht in die jeweiligen Möglichkeiten und Wege zu optimaler medizinischer und menschlicher Betreuung zugestanden wird.

Dr. med. Armin Oberle, Lenzburg 


\section{Changement d'éthique}

Durant 25 siècles, le rôle premier sinon exclusif du médecin a été de soigner le malade selon des principes dits hippocratiques qui focalisaient toute la pratique de la médecine sur ce dernier. D'où l'éthique médicale, cette quintessence de la morale du médecin en exercice, qu'il promettait d'observer en prononçant solennellement le serment d'Hippocrate, résumé des principes promouvant et justifiant l'empathic, le dévouement et les sacrifices d'une profession dont la pénibilité et les exigences de disponibilité et de formation nécessitaient une réelle «vocation». Sur le plan juridique, cette relation singulière (colloquium singulare) médecin - malade dépendait du contrat de mandant réglant les rapports de deux êtres également libres et donc indépendants et responsables.

En fait, cette éthique médicale (hippocratique) consistait à soigner le malade selon les besoins propres de ce dernier à l'encontre, comparativement, de l'éthique vétérinaire qui consiste à soigner la bête malade, avant tout selon les intérêts et les impératifs de son propriétaire, employeur du vétérinaire et débiteur du coût des soins fournis. Mais depuis une cinquantaine d'années, l'évolution collectiviste de la société a changé progressivement la polarité médicale. Dès 1964, le socialiste Tschudi indiquait la voie: «Le médecin doit être au service des caisses-maladie». En 1967, le TFA par l'arrêté Streicher «légalisait» cette direction qui permit, par l'intermédiaire des caisses, d'étatiser progressivement la médecine, d'en détruire les principes hippocratiques et de dynamiser la parabolique courbe des coûts de la santé.
Aujourd'hui, le médecin n'est plus qu'un instrument du pouvoir. On en a fait le simple outil d'une politique de santé axée sur la systémique des caisses-maladie et en concurrence avec les autres dicastères (routes, armée, etc.) de l'Etat. De cette politique et de ce pouvoir pour lesquels la vie et la survie de tel ou tel individu malade ne saurait évidemment qu'être un épiphénomène, un détail sujet, éventuellement et au mieux, à statistique. Son rôle actuel est d'appliquer la politique de santé en veillant à l'économicité de celle-ci afin de sauvegarder le statut et le standing de ses gestionnaires. Autrement dit d'être le chien de garde du capital, accumulé par les cotisations des assurés et les impôts (subventions) des contribuables, que le coût des soins nécessités par les malades agresse et pourrait mettre en péril.

Il a perdu toute indépendance dans l'exercice de sa profession. Il est devenu le pantin des bureaucraties ignares et envieusement complexées des caisses et de l'Etat, un sous-citoyen incapable d'offrir au patient, l'aval discrétionnaires de celles-ci, les compétences qu'il s'est forgées durant de longues années. Il n'aura bientôt même plus le choix du traitement à appliquer, c'est tout dire! Le système lui a fait perdre son âme et son crédit et par la même, la confiance des populations qui considèrent le médecin comme un simple «fournisseur» étatique de soins, à consommer à gogo, aux frais de la collectivité.

Le prestige narcissique du «grand patron» a fait place à l'obtuse arrogance des «golden boys» des caisses-maladie. Est-ce vraiment un progrès pour le malade?

Dr. E. Truffer, Sierre 
解

Ärztestopp, Notfalldienst, Nachfolgeprobleme

Mit der Einführung des umstrittenen Ärztestopps kommt es in nichtstädtischen Gegenden zu einem Problem, das von den entsprechenden Stellen wahrscheinlich nicht erkannt worden ist. Die jetzige Situation führt zu einer Überalterung der Grundversorger. Diese sind ab ihrem 60. Altersjahr von der Notfalldienstpflicht befreit. Für die jüngeren Kollegen kann das bedeuten, dass die Anzahl der Notfalldienste um 25 bis $50 \%$ erhöht wird. In unserer Gemeinde heisst dies beispielsweise 73 Diensttage statt 40 pro Jahr. Dies ist eine massive Einschränkung der Lebensqualität.

Von älteren Ärzten, die bereit wären, weniger zu arbeiten oder ihre Praxis zu übergeben, hört man immer wieder, dass sie grosse Probleme haben, Nachfolger/innen zu finden. Sehr oft kommen Anfragen von Ärzten aus dem EU-Raum, die bereit wären, als Grundversorger tätig zu sein. Dies sollte dann aber auch nicht das Ziel unserer Bemühungen sein.

Aus dem Engpass, in den wir hineinmanövriert werden, kommen wir nur noch heraus, wenn die Dienstpflicht für alle Grundversorger/innen erhalten bleibt, solange sie eine Praxisbewilligung haben und eine geregelte Tätigkeit ausüben. Zudem sollte der Anreiz, als Grundversorger/in tätig zu sein, vermehrt Studierenden und Assistenzärzten/-ärztinnen bekanntgemacht werden, so dass sich junge Kolleginnen und Kollegen freuen, diesen Beruf ausüben zu dürfen. Individuelle Lösungen mit fliessenden Übergaben oder Praxisgemeinschaften sind auszuloten und könnten der schwierigen Situation, die auf uns zukommt, entgegenwirken.

Es dürfte nicht Sinn dieser Bestimmungen sein, dass niedergelassene Ärztinnen und Ärzte durch Überbelastung ihrer physischen und psychischen Kräfte schädigende Auswirkungen auf Gesundheit und Leistungsfähigkeit erleiden. Neue Ideen und Erlasse zur temporären Überbrückung der Situation sind gefragt.

Dr. med. Bernhard Sorg, Wallisellen

\section{Ärztliche Notfalldienstplanung mit dem Internet}

Als betroffener Grundversorger im nicht grossstädtischen Zentrum, sondern in der Agglomeration, kann die Lobpreisung von «docbox» nicht unwidersprochen bleiben [1]. Mag sein, dass in der Stadt Zürich das System erfolgreich ist (ich kann es nicht beurteilen), im Raum Dietikon (unteres Limmattal) bleibt es nach wie vor höchst kontrovers. Ein Teil der beteiligten Ärzte hatte letzten November einen Ausstieg aus dem System vorgeschlagen. In der Notfalldienstsitzung wurde letztlich beschlossen, «docbox» nochmals ein Jahr lang eine Chance zu geben. Sollten die notwendigen Verbesserungen und Anpassungen nicht erfolgen oder nicht möglich sein, wird erneut über einen Ausstieg abgestimmt. Insgesamt ist der Aufwand sowohl für die Eingabe der Abwesenheiten vor Dienstplanung als auch für Diensttausch um ein Vielfaches höher als zuvor in der nichtelektronischen Form. Ausserdem zeigte der elektronisch erstellte Dienstplan groteske Dienstverteilungen, wie sie kein menschliches Auge zulassen würde.

Hinzu kommt die plötzliche Einführung «von oben herab», das heisst, kurz vor der Dienstplanung wurden die Ärzte im Dienstkreis unteres Limmattal im Jahr 2002 darüber informiert, dass die Planung jetzt elektronisch erfolge. Die «Basis» wurde nicht konsultiert, ob sie das denn auch gut und sinnvoll finde (schliesslich kostet das Ganze ja auch etwas...).

Ich empfehle allen Ärzten, die sich einen Wechsel auf dieses (oder überhaupt ein elektronisches) System überlegen, sich zuvor ein Bild davon zu machen, was das für ihre konkrete Situation und Region bedeutet.

Dr. med. O. Dätwyler, Dietikon

1 Ronalter D, Castelberg U, Willi O. Ärztliche Notfalldienstplanung mit dem Internet. Schweiz Ärztezeitung 2004;85(10):509-11. 